\title{
AVALIAÇÃO DA CINÉTICA DE MOAGEM DO MINÉRIO DA JACOBINA MINERAÇÃO E COMÉRCIO
}

\author{
L. R. P. DE ANDRADE LIMA* e J. N. NUNES \\ Departamento de Ciência e Tecnologia dos Materiais - Universidade Federal da Bahia \\ lelo@ufba.br*
}

Artigo submetido em novembro/2013 e aceito em outubro/2014

DOI: $10.15628 /$ holos.2014.1831

\section{RESUMO}

O minério aurífero da Jacobina Mineração e Comércio foi amostrado e usado para a determinação das funções de seleção $S$ e de quebra $B$, usando moagem descontinua de nove faixas granulométricas entre 2000 e 75 micra. Foi observado que a taxa de desaparecimento segue o modelo de primeira ordem e a função de seleção possui o clássico comportamento de aumento proporcional ao tamanho das partículas seguido por uma redução do valor para partículas grandes. A função de quebra tem um comportamento normalizável. As funções puderam ser ajustadas com os seguintes modelos:

$$
S_{i}=\frac{0,86 d_{i}^{1,14}}{1+\left(\frac{d_{i}}{1,56}\right)^{4,67}}
$$

$$
B_{i, j}=0,29\left(\frac{d_{i-1}}{d_{j}}\right)^{0,76}+0,71\left(\frac{d_{i-1}}{d_{j}}\right)^{2,21}
$$

PALAVRAS-CHAVE: moagem; modelo matemático; minério de ouro.

\section{EVALUATION OF THE GRINDING KINETICS TO THE ORE FROM THE JACOBINA MINERAÇÃO E COMÉRCIO}

\begin{abstract}
The Jacobina Mineração e Comércio gold ore was sampled and used to evaluate the selection $\mathrm{S}$ and breakage $B$ functions using batch grinding experiments and nine size fractions between 2000 and 75 micron. The grinding rate follows the first order model and the selection function presents the classical behavior of enhancement with the particle size followed by a reduction for large particles size. The breakage function could be normalized. The grinding functions $S$ and $B$ could by fit with the models:
\end{abstract}

$$
S_{i}=\frac{0,86 d_{i}^{1,14}}{1+\left(\frac{d_{i}}{1,56}\right)^{4,67}}
$$

and

$B_{i, j}=0,29\left(\frac{d_{i-1}}{d_{j}}\right)^{0,76}+0,71\left(\frac{d_{i-1}}{d_{j}}\right)^{2,21}$

KEYWORDS: grinding; mathematical model; gold ore. 


\section{INTRODUÇÃO}

A modelagem e a simulação de circuitos de moagem de minérios tem sido objeto de estudos por mais de 50 anos e atualmente é uma ferramenta indispensável para o projeto e para a otimização sistemática de usinas de beneficiamento, sobretudo devido ao fato que a cominuição corresponde a uma importante parcela do custo total destas unidades industriais. Na usina da Jacobina, por exemplo, o custo da moagem corresponde a cerca de $50 \%$ do custo operacional da usina, do qual 30\%, o que não esta relacionado ao consumo de corpos moedores, pode ser reduzido pela otimização do circuito de moagem-classificação.

Os modelos fenomenológicos usados para descrever a moagem de minérios tiveram origem nos 1950. BASS (1954) expressa uma teoria matemática e um modelo fenomenológico para a moagem descontinua, que resultou na equação diferencial que descreve a evolução temporal da distribuição granulométrica das partículas $(F(d, t))$ durante a moagem:

$\frac{\partial^{2} F(d, t)}{\partial d \partial t}=-S(d) \frac{\partial F(d, t)}{\partial d}+\int_{d}^{d_{0}} \frac{\partial B(d, \alpha)}{\partial d} S(\alpha) \frac{\partial F(\alpha, t)}{\partial d} d \alpha$

em que d é o tamanho das partículas, do é o tamanho inicial das partículas, t é o tempo de moagem, F é a frequência acumulada passante, $S$ é a taxa de quebra das partículas (ou função de seleção) e B é a função de quebra das partículas. A Equação (1) é conhecida com equação fundamental da cominuição e tem soluções analíticas apenas para casos muito particulares o que limitou a sua ampla utilização por vários anos.

Na Equação (1) a cinética de fragmentação das partículas do minério de dimensão d é descrita pela função de seleção $(S(d))$. A fragmentação de minérios em geral segue uma cinética de primeira ordem, sendo válida a equação (AUSTIN et al., 1984; AUSTIN e CONCHA, 1993):

$$
\frac{d M(d, t)}{d t}=-S(d) M(d, t)
$$

onde $\mathrm{M}$ é a massa de partículas de tamanho d. Incluindo a distribuição granulométrica do minério, a Equação (2) pode ser re-escrita como:

$$
\frac{d\left[M_{\text {Total }} f(d, t)\right]}{d t}=-S(d)\left[M_{\text {Total }} f(d, t)\right]
$$

em que MTotal é a massa total do minério e f é a frequência simples das partículas de dimensão $d$. Para a moagem descontinua o sistema é fechado, portanto a massa total é constante e a equação resultante pode ser simplificada:

$\frac{d f(d, t)}{d t}=-S(d) f(d, t)$

A integração da Equação (4) para o caso em que a distribuição de tamanhos inicial $f(d, 0)$ é conhecida e resulta em:

$$
f(d, t)=f(d, 0) e^{-S(d) t}
$$

A discretização da Equação (5) permite obter: 


$$
f_{i}(t)=f_{i}(0) e^{-S_{i} t}
$$

em que $f_{i}$ é a frequência simples da distribuição granulométrica das partículas de diâmetro $d_{i}$ e $S_{i}$ é a correspondente taxa de desaparecimento das partículas da faixa granulométrica i. Portanto, utilizando-se a moagem em faixas granulométricas estreitas, o gráfico semi-log para cada tamanho produz retas, caso a hipótese de cinética de primeira ordem seja atendida, o que não se aplica no caso de materiais quebradiços. As inclinações destas retas fornecem os valores estimados das velocidades específicas de quebra do minério para cada faixa de tamanho $\left(\mathrm{S}_{\mathrm{i}}\right)$.

REID (1965) propôs a discretização da Equação (1) como uma aproximação prática, uma vez que a forma corrente de análise das distribuições granulométricas de minério uma série de peneiras padronizadas, o que permite inferir a distribuição de tamanhos real das partículas. A versão discretizada da Equação (1) tem a seguinte forma:

$$
\frac{d f_{i}(t)}{d t}=-S_{i} f_{i}(t)+\sum_{\substack{j=1 \\ i>j}}^{i-1} b_{i, j} S_{j} f_{j}(t) \quad n \geq i \geq j \geq 1
$$

em que $f_{i}$ é a frequência simples da classe granulométrica i, t é o tempo de moagem, $S_{i}$ é a taxa de quebra do material (ou função de seleção) da classe granulométrica i e $b_{i, j}$ é a função de quebra (na forma não-acumulada, $b_{i, j}=B_{i, j}-B_{i+1, j}$ ) das partículas do minério da classe granulométrica $i$ gerando a classe granulométrica j. A Equação (7) é similar a Equação (4), mas além do desaparecimentos das partículas da classe granulométrica i, leva em consideração a formação das partículas nesta classe oriundas da fragmentação das partículas maiores.

O conjunto de $n$ equações diferenciais ordinárias, Equação (7), que fornece a evolução temporal da distribuição granulométrica das partículas do minério durante a moagem descontinua foi resolvido analiticamente por REID (1965), resultando nas seguintes equações:

$$
f_{i}(t)=\sum_{j=1}^{i} a_{i, j} e^{-S_{j} t}
$$

em que,

$$
\begin{aligned}
& a_{i, j}=f_{i}(0)-\sum_{\substack{k=1 \\
i>j}}^{i-1} a_{i, k} \quad, \quad i=j \\
& a_{i, j}=\frac{1}{S_{i}-S_{j}} \sum_{k=j}^{i-1} S_{k} b_{i, k} a_{i, k} \quad, \quad i>j
\end{aligned}
$$

Outras soluções para a Equação (7), assim como a versão para o caso de moagem contínua são encontradas na literatura (AUSTIN et al., 1984; BERALDO, 1987; AUSTIN e CONCHA, 1993), mas fogem ao escopo deste trabalho.

A determinação dos parâmetros cinéticos da moagem (funções $S_{i}$ e $B_{i, j}$ ), característicos do mineral e das condições de ensaio podem ser feitas por retrocálculo, quando há disponibilidades de informação da unidade de moagem, ou pelos ensaios de moagem e classificação em laboratório, como é o caso deste trabalho (AUSTIN et al., 1984; AUSTIN e CONCHA, 1993).

A função de seleção pode ser estimada pela moagem de partículas de faixas granulométricas estritas em diferentes tempos e a determinação da inclinação das curvas de $f(t)$ linearizadas, como indica a Equação (7). A função $S$ depende do tamanho das partículas do minério 
e da razão diâmetro dos corpos moedores do moinho versus diâmetro de partícula. Nota-se que os valores de $\mathrm{S}$ crescem com o aumento do tamanho das partículas, mas depois de certo tamanho de partículas a função atinge um valor máximo e, em seguida, esta tendência é invertida (AUSTIN et al., 1984; AUSTIN e CONCHA, 1993). Algumas equações foram propostas para descrever a função S, uma delas é a equação proposta por AUSTIN et al. (1984), que consegue descrever muitos casos:

$$
S_{i}=\frac{\alpha_{0} d_{i}^{\alpha_{1}}}{1+\left(\frac{d_{i}}{d_{\text {crit }}}\right)^{\alpha_{2}}} \quad, \quad i=1,2, \ldots, n
$$

em que, $\alpha_{0}, \alpha_{1}, \alpha_{2}$ e $d_{\text {crit }}$ são os parâmetros ajustáveis do modelo.

A função quebra B pode ser estimada por meio de ensaios de moagem por curto período de tempo para uma classe de partículas de minério com faixas granulométricas estreitas. Este procedimento evita a fragmentação secundaria das partículas, o que levaria a valores incorretos da função B. Deve ser assegurado que menos de $30 \%$ das partículas de cada faixa granulométrica sejam fragmentadas neste procedimento (AUSTIN e LUCKIE, 1972; AUSTIN et al., 1984). Neste caso se o maior tamanho é j a função de quebra é dada pela Equação (12):

$$
B_{i, j} \approx \frac{\log \left[\frac{1-F_{i}(0)}{1-F_{i}(t)}\right]}{\log \left[\frac{1-F_{j+1}(0)}{1-F_{j+1}(t)}\right]} \quad, \quad i>j
$$

Nota-se que os valores de $B_{i, j}$ não devem ser influenciados pelas condições de moagem, tais como carga do minério, carga de bolas, diâmetro do moinho, dentre outros. Nota-se também que para a maior parte dos minérios os valores de $B_{i, j}$ são normalizáveis, ou seja uma vez que as partículas se quebram com uma distribuição dimensionalmente similar. Por esta razão, na maior parte dos casos, os valores de $\mathrm{B}_{\mathrm{ij}}$ podem ser ajustados por uma relação empírica, que é composta pela soma de duas funções lineares em escala log-log (AUSTIN et al., 1984; AUSTIN e CONCHA, 1993):

$$
B_{i, j}=\Phi\left(\frac{d_{i-1}}{d_{j}}\right)^{\gamma}+(1-\Phi)\left(\frac{d_{i-1}}{d_{j}}\right)^{\beta} \quad, \quad i>j
$$

em que $\Phi, \gamma$ e $\beta$ são os parâmetros ajustáveis do modelo e são característicos do minério. Os valores de $\Phi$, estão entre 0 e $1, \gamma$ estão entre 0,5 e 1,5 e de $\beta$ estão tipicamente na faixa de 2,5 a 5,0. A função da Equação (13) o também é chamada de função distribuição da quebra normalizada (AUSTIN et al., 1984).

A simulação da moagem contínua pode ser feita levando em consideração a função de quebra do minério (B), determinada usando ensaios de moagem descontinua, a função de seleção (S), determinada para o moinho continuo por retrocálculo e a função distribuição dos tempos de residência do moinho continuo $(E(t))$, através da equação (AUSTIN et al., 1984; AUSTIN e CONCHA, 1993): 


$$
f_{p}=\left[\int_{0}^{\infty} e^{-(B-I) S t} E(t) d t\right] f_{a}
$$

em que $f_{a}$ é o vetor com a freqüência simples das partículas do minério da alimentação e $f_{p}$ é o vetor com a freqüência simples das partículas do minério do produto da moagem. A função distribuição dos tempos de residência pode ser facilmente estimada através de ensaio com traçador. Freqüentemente moinhos industriais podem ser descritos como uma cascata de misturadores perfeitos com um grande em serie com dois pequenos de igual tamanho (AUSTIN et al., 1984; AUSTIN e CONCHA, 1993):

$E(t)=\frac{1}{\tau_{1}-\tau_{2}}\left[\frac{t}{\tau_{1}} e^{-\frac{t}{\tau_{1}}}+\frac{\tau_{2}}{\tau_{2}-\tau_{1}}\left(e^{-\frac{t}{\tau_{1}}}-e^{-\frac{t}{\tau_{2}}}\right)\right]$

em que $\tau_{1}$ é o tempo médio de residência do reator grande e $\tau_{2}$ é o tempo médio de residência dos reatores pequenos.

Neste trabalho estuou-se um minério de ouro da Jacobina Mineração e Comércio Ltda (JMC), por conseguinte, foram realizados ensaios de moagem e classificação do minério com o objetivo de determinar os parâmetros das funções de quebra $\left(B_{i, j}\right)$ e de seleção $\left(S_{i}\right)$, os quais são necessários para a caracterização do minério.

\section{MATERIAIS E MÉTODOS}

A amostra, utilizada nos ensaios, foi homogeneizado e re-amostrada usando um amostrador Jones da Rodoteste. Os ensaios de moagem foram conduzidos em um moinho de bolas de laboratório de aço com 19,2 cm de diâmetro interno e 20,5 cm, carregado com 72 bolas de aço de 2,46 cm de diâmetro e 16 bolas de 4,08 cm de diâmetro. A velocidade de rotação usada foi de aproximadamente 70 RPM. Após cada ensaio de moagem o material foi classificado em um Ro-tap da Marconi modelo MA750 usando uma serie de peneiras com as seguintes aberturas: 2000, 1180, 850, 600, 500, 300, 250, 150, 106 e $75 \mu \mathrm{m}$ (ou seja 10, 16, 20, 30, 35, 50, 60, 100, 140 e 200 malhas $A B N T)$. A massa de minério retida em cada faixa granulométrica foi pesada em uma balança Shimadzu modelo BL3200H.

Para preparação das amostras e determinação dos parâmetros das funções seleção (S) e quebra (B) foram realizados ensaios descontínuos de moagem no moinho de bolas em intervalos de tempo de: um, dois, quatro e sete minutos. O primeiro intervalo, um minuto, foi necessário para permitir estimar os parâmetros da função quebra (B) e, para os demais, foram escolhidos de maneira a caracterizar adequadamente a taxa de desaparecimento das faixas granulométricas de maneira a permitir a estimativa precisa da função seleção (S).

\section{RESULTADOS E DISCUSSÕES}

Na Figura 1 observa-se a evolução da frequência acumulada passante durante a moagem das nove faixas granulométricas do minério considerados neste estudo. Foram considerados os intervalos de moagem de um, dois, quatro e sete minutos. Ao usar a frequência acumulada retida para cada classe granulométrica em função do tempo e a Equação (5) foi possível estimar a cinética de quebra de cada classe de tamanho. Observa-se na Figura 2 que, para todas as frações 
granulométricas consideradas, o minério da JMC segue uma cinética de fragmentação de primeira ordem.

Neste caso, a taxa de fragmentação aumenta com o aumento do tamanho das partículas, mas para partículas muito grandes esta taxa diminui, conforme descrito por AUSTIN e CONCHA (1993). A velocidade de quebra em função do diâmetro médio das faixas granulométricas usadas, assim como, o modelo ajustado usando o método dos mínimos quadrados, podem ser visualizados no gráfico da Figura 3.
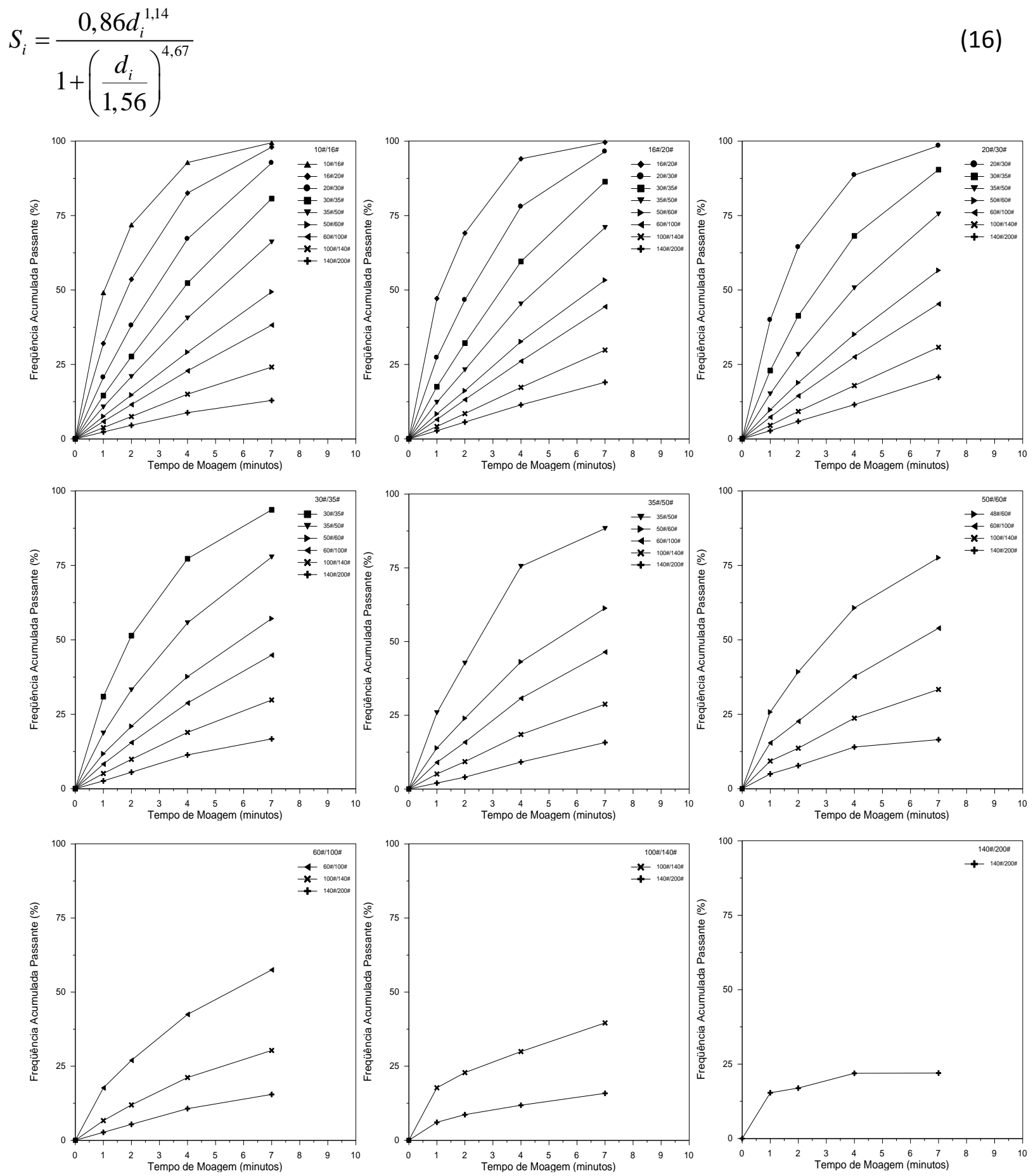

Figura 1 - Evolução temporal da moagem de mano-tamanhos. 


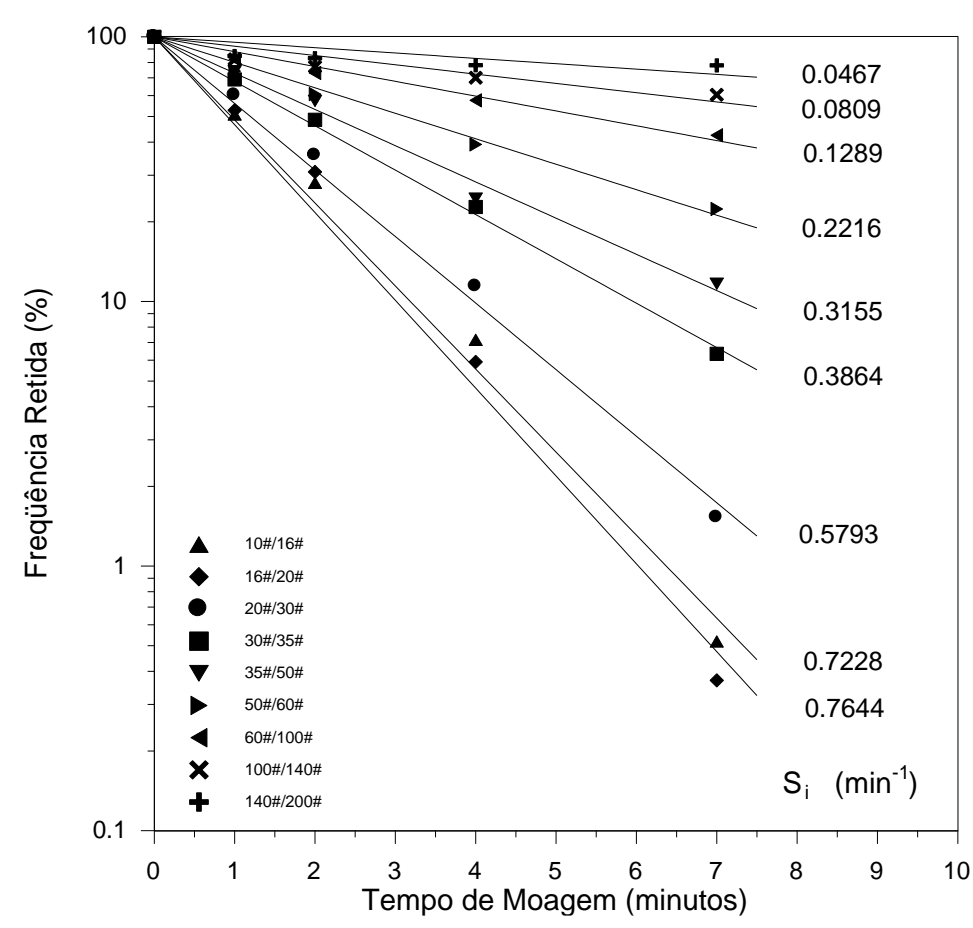

Figura 2 - Cinética da moagem em função do tamanho das partículas.

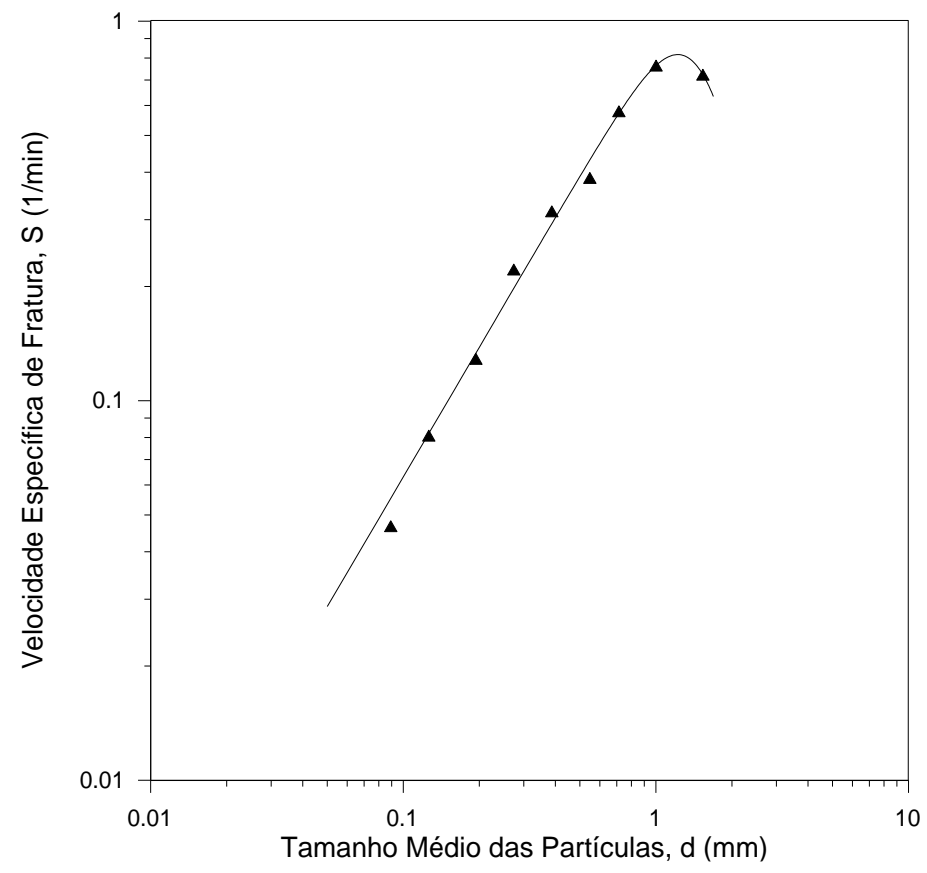

Figura 3 - Velocidade específica de quebra das partículas em função do tamanho.

Ao usar o tempo de um minuto de moagem para as nove faixas granulométricas e a Equação (13), foi possível estimar a função de quebra (B) do minério da JMC. Na Tabela 1 sumariza-se os resultados da função quebra (b) e da função quebra acumulada (B) para a fração granulométrica mais grossa. Os valores da função quebra acumulada para as nove faixas granulométricas consideradas neste estudo exibidos na Figura 4. Nota-se que há alguma dispersão, mas há uma tendência a normalização dos resultados. Na Figura 4 também exibe-se o modelo ajustado para todos os dados, usando o método dos mínimos quadrados: 
$B_{i, j}=0,29\left(\frac{d_{i-1}}{d_{j}}\right)^{0,76}+0,71\left(\frac{d_{i-1}}{d_{j}}\right)^{2,21}$

Tabela 1 - Função quebra (b) e função quebra acumulada (B) para as partículas de 10/16 malhas ABNT

\begin{tabular}{ccccc}
\hline Numero do Intervalo & $\begin{array}{c}\text { Tamanho ABNT } \\
\text { (malha) }\end{array}$ & $\begin{array}{c}\text { Abertura } \\
(\mu \mathrm{m})\end{array}$ & $\mathrm{B}_{\mathrm{i} 1}$ & $\mathrm{~b}_{\mathrm{i} 1}$ \\
\hline 1 & $10 / 16$ & $2000 / 1180$ & 1,00 & \\
2 & $16 / 20$ & $1180 / 850$ & 0,57 & 0,23 \\
3 & $20 / 30$ & $850 / 600$ & 0,34 & 0,11 \\
4 & $30 / 35$ & $600 / 500$ & 0,23 & 0,07 \\
5 & $35 / 50$ & $500 / 300$ & 0,17 & 0,05 \\
6 & $50 / 60$ & $300 / 250$ & 0,12 & 0,03 \\
7 & $60 / 100$ & $250 / 150$ & 0,09 & 0,03 \\
9 & $100 / 140$ & $150 / 106$ & 0,06 & 0,02 \\
\hline
\end{tabular}

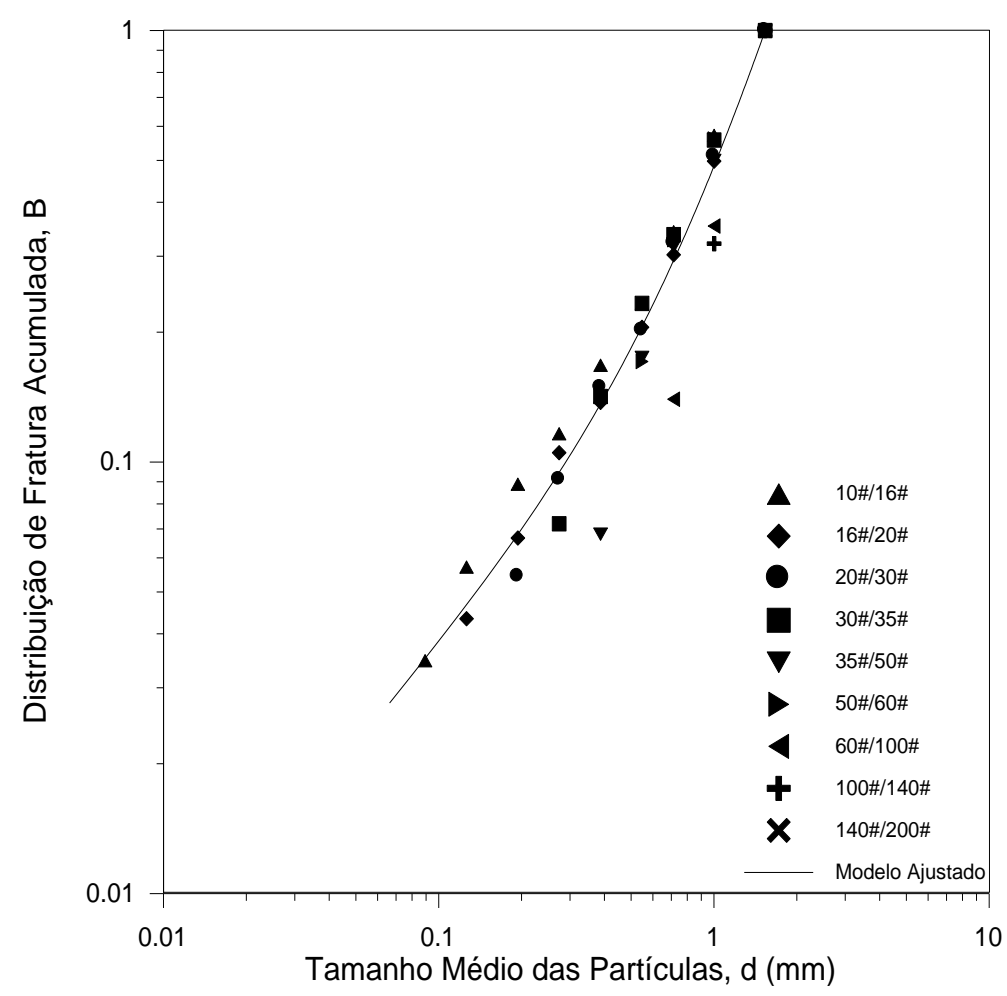

Figura 4 - Função quebra acumulada em função do tamanho das partículas.

\section{CONCLUSÕES}

O minério aurífero da Jacobina Mineração e Comércio foi amostrado e usado para a determinação das funções de seleção $\mathrm{S}$ e de quebra B usando moagem descontinua de nove faixas granulométricas entre 2000 e $75 \mu \mathrm{m}$.

A taxa de desaparecimento segue o modelo de primeira ordem e a função de seleção corresponde ao clássico comportamento de aumento com o aumento do tamanho das partículas seguido por uma redução do valor para partículas grandes. A função de quebra tem um 
comportamento normalizável. Ambas as funções puderam ser bem ajustadas com modelos empíricos previamente descritos na literatura.

\section{AGRADECIMENTOS}

Os autores agradecem o apoio da Jacobina Mineração e Comércio e ao PIBIC-UFBA por conceder uma bolsa de Iniciação Cientifica para este projeto.

\section{REFERÊNCIAS BIBLIOGRÁFICAS}

1. AUSTIN, L.G., LUCKIE, P.T., Methods for determination of breakage distribution parameters, Powder Technology, v.5, n.4, p. 215-222, 1972.

2. AUSTIN, L.G., KLIMPEL, R.R., LUCKIE, P.T., Process Engineering of Size Reduction: Ball Milling, Society of Mining Engineers of the American Institute of Mining, Metallurgical, and Petroleum Engineers, 1984.

3. AUSTIN, L.G., CONCHA, F., Diseño y simulación de circuitos de molyenda y classificacion, CYTED. 1993.

4. BASS, L., Zur theorie der mahlvorgange, Z. angew. Math. Phys., v.5, p.283-292 , 1954.

5. BERALDO, J.L., Moagem de Minérios em Moinhos Tubulares, Edgard Blucher Ltda, 1987.

6. REID, K.J., A solution to the batch grinding equation, Chemical Engineering Science, v.20, n.11, p.953-963, 1965. 